Trabajos Originales

\title{
Glicosaminoglicanos como posibles reguladores de inflamación durante la malaria placentaria
}

\author{
Erika Garrido Z.1a \\ ${ }^{1}$ Grupo de Investigación en Salud Familiar y Comunitaria (GISAFACO). Programa de Medicina. Facultad de Ciencias de \\ la Salud. Corporación Universitaria Remington. Medellín, Colombia.
}

a Microbióloga, MSc Biología.

\section{RESUMEN}

Los mecanismos fisiopatológicos de la malaria placentaria son hasta el momento poco comprendidos, y el daño placentario derivado de la infección por Plasmodium spp se ha relacionado con eventos adversos del embarazo que afectan directamente el desarrollo del feto. Las concentraciones placentarias de algunas citocinas como la IL-10, TNF- $\alpha$ y TGF- $\beta$ y glicosaminoglicanos como el CSA, HA y HS podrían estar participando de forma reguladora en los eventos inflamatorios placentarios durante la infección por Plasmodium spp.

\section{PALABRAS CLAVE: Malaria placentaria, inflamación placentaria, citosinas, placenta, glicosaminoglicanos}

\section{SUMMARY}

The pathophysiological mechanisms of placental malaria are until now poorly understood and the placental damage resulting from infection by Plasmodium spp has been linked to adverse pregnancy events that directly affect fetal development. Placental concentrations of some cytokines such as IL-10, TNF- $\alpha$ and TGF- $\beta$ and glycosaminoglycans such as CSA, HA and HS could be involved in a regulatory role in placental inflammation during infection by Plasmodium spp.

\section{KEY WORDS: Placental malaria, placental inflamation, cytokines, placenta, glycosaminoglycans}

\section{INTRODUCCIÓN}

En áreas de transmisión para la malaria, después de los niños menores de cinco años, las gestantes son la población más susceptible para contraer la infección, debido tanto a los cambios hormonales como a las variaciones en la respuesta inmune (1), que aún siendo condiciones fisiológicas propias del embarazo, predisponen a la gestante a un mayor riesgo frente a las infecciones. La susceptibilidad es aún mayor en mujeres nunca antes expuestas a la malaria, debido principalmente a la falta de patrones inmunitarios frente a la presencia del parásito, que se construyen tras sucesivos episodios de malaria.

Para evadir la respuesta inmune, Plasmodium spp hace que las formas maduras del parásito se secuestren en los lechos vasculares de órganos 
profundos, donde ha encontrado receptores de adherencia para los glóbulos rojos parasitados; en el caso de las gestantes, el parásito genera fenotipos adherentes que reconocen al condroitín sulfato $A$ (CSA) como receptor de adherencia, el CSA, se halla exclusivamente en la placenta, lo que explica que los parásitos placentarios no se aíslen en ningún otro individuo no gestante, por lo tanto, la presencia de CSA en la placenta es otro factor predisponente para el padecimiento de la infección placentaria (2). En la placenta, se encuentran otros glicanos como el heparán sulfato (HS) y el ácido hialurónico (HA) que forman parte de la matriz extracelular de dicho tejido y que además de receptores, podrían tener participación en eventos regulatorios de la respuesta inmunitaria local $(3,4)$.

La presencia de parásitos de la malaria en la placenta y de hemozoina, inducen la movilización de células fagocíticas como monocitos/macrófagos y neutrófilos hacia el sitio de la infección y estas a su vez liberan citocinas proinflamatorias que inducen alteraciones patológicas de la placenta impidiendo un adecuado intercambio de oxígeno y nutrientes en la interface materno-fetal $(5,6)$, por tal razón, la malaria placentaria se ha asociado con eventos adversos del embarazo que comprometen la salud de la gestante y el desarrollo del feto (7).

\section{Agente causal y ciclo de vida}

La malaria placentaria (MP) es una infección causada por parásitos del género Plasmodium, pertenecientes al Phylum Apicomplexa. Hasta la fecha se han documentado 5 especies responsables de la infección a humanos, P. falciparum, P. vivax, P. ovale, P. malariae y P. knowlesi. El primero es la especie más ampliamente estudiada y se considera la más agresiva y el segundo es la especie prevalente en Latinoamérica.

Los parásitos de la malaria son vectorizados por mosquitos hembra del género Anopheles. En el vector, el parásito desarrolla la fase sexuada o gametogonia y en el hospedero intermediario (vertebrado - hombre) lleva a cabo la etapa asexuada denominada esquizogonia; de igual forma, en el vertebrado presenta un ciclo hepático y un ciclo intraeritrocítico.

\section{Susceptibilidad de la gestante y la placenta a la malaria}

Existen factores que predisponen a la gestante a un mayor riesgo de padecer la infección malárica si se compara con su contraparte no gestante. La embarazada experimenta cambios fisiológicos como una mayor temperatura corporal con la consecuente sudoración, y por ende, una mayor emisión de sustancias volátiles producto de la actividad de su microflora. Dichos cambios fisiológicos, además de una mayor emisión de dióxido de carbono, se constituyen en señales quimio atrayentes para el mosquito, aumentando la probabilidad de la picadura (8).

Otro elemento que pone a las gestantes como un grupo de mayor riesgo frente a la malaria, es la modulación de la respuesta inmune que tiene lugar durante la gestación, pues debido a la presencia de antígenos paternos en el feto, este podría ser rechazado como un aloijerto. Para promover el sostenimiento de la gestación, y lograr esa modulación inmune, la embarazada presenta niveles elevados de estrógenos y progesterona que ejercen su efecto directamente sobre una subpoblación de células NK (natural killer) placentarias con actividad lítica reducida y sobre las células de la decidua inhibiendo en estas la liberación de citocinas proinflamatorias. La madre por lo tanto exhibe un perfil inmune basado principalmente en una respuesta de tipo humoral quedando expuesta a los agentes microbianos intracelulares como Plasmodium que requieren una respuesta inmune de tipo celular (9-11).

Una vez infectada la gestante, los parásitos presentan una gran afinidad para adherirse al epitelio de las vellosidades trofoblásticas, pues la población de parásitos de gestantes expresa una variante de la proteína de membrana del eritrocito de Plasmodium falciparum 1 (PfEMP1), exclusiva de las embarazadas, que tiene como receptor de adherencia al CSA, glicano abundante en la placenta y no accesible para esta población parasitaria particular en otro lecho vascular (12), frente al que se desarrollan anticuerpos tras sucesivas infecciónes en gestaciones diferentes $(13,14)$. La presencia del parásito induce un cambio del estado inmunológico por lo que se activa en la placenta una marcada respuesta tipo Th1 o celular, promoviendose la liberación de citocinas proinflamatorias como el TNF- $\alpha$, IFN-Y, IL-8, entre otras. La elevada expresión de estas citocinas a nivel local, es responsable del daño tisular que se ha observado en las placentas maláricas y, como consecuencia de ello, del impacto de la malaria placentaria sobre el desarrollo del feto (15-17).

\section{Respuesta inflamatoria en la placenta}

En la placenta malárica, el microambiente inflamatorio es alterado por la presencia del parásito, al igual que en otras infecciones intracelulares como leishmaniasis, toxoplasmosis y tuberculosis, el perfil 
de citocinas en la placenta se inclina hacia un predominio Th1, es decir, citocinas proinflamatorias (IL-2, IFN-Y y TNF- $\alpha$ ); en cuanto al perfil Th2 (IL-10, IL-6 y TGF- $\beta$ ) citocinas antiinflamatorias o reguladoras, los resultados son discrepantes, no dejando claro el rol antiinflamatorio de la IL-10 y el TGF- $\beta$.

Se ha descrito ya que como consecuencia de la implantación y la placentación, se genera un ambiente inflamatorio en la placenta, puesto que las NKu y células del trofoblasto bajo influjo hormonal, son activas en la liberación de citocinas proinflamatorias que inducen un reclutamiento de diferentes células inmunes como linfocitos, células dendríticas, monocitos/macrófagos y neutrófilos que tienen a cargo importantes funciones para el sostenimiento de la gestación y durante el parto (18). En pequeñas cantidades, las citocinas proinflamatorias cumplen con las funciones ya descritas; no obstante, durante la infección por Plasmodium spp, se ha encontrado un aumento de las mismas que produce daño de la arquitectura placentaria y este a su vez genera efectos adversos en el desarrollo del feto y en la salud de la gestante (19).

\section{Histopatología de la placenta}

Durante la infección por $P$. falciparum la placenta puede acoger una gran cantidad de parásitos, células inflamatorias y pigmento malárico. Estos hallazgos fueron descritos inicialmente por Bignami en 1898, y Serini en 1902 (20), posteriormente, se reportó la presencia de parásitos y pigmento en extendido delgado de sangre placentaria, la marcada susceptibilidad de la placenta a la infección malari$\mathrm{ca}$, depende en gran medida de mayores niveles de cortisol presentes en las gestantes con malaria y la modulación inmune local (16).

Al examen histológico, tanto los parásitos como la hemozoina son detectados fácilmente y cuando hay presencia de parásitos en el tejido, se localizan en el EIV; por su parte, el pigmento, se distribuye libre, sobre depósitos de fibrina de la placenta o al interior de macrófagos que se reclutan en mayores cantidades en la placenta durante la infección por P. falciparum. Una característica llamativa del secuestro placentario es la ausencia de rosetas que sí se observa en la malaria cerebral, lo que probablemente se debe al fenotipo de adherencia de los parásitos que infectan a la placenta (21) (Figura 1).

Los estudios que documentan el daño placentario como consecuencia de la infección por parásitos de la malaria, han descrito diversas lesiones que se presentan debido a un dramático aumento

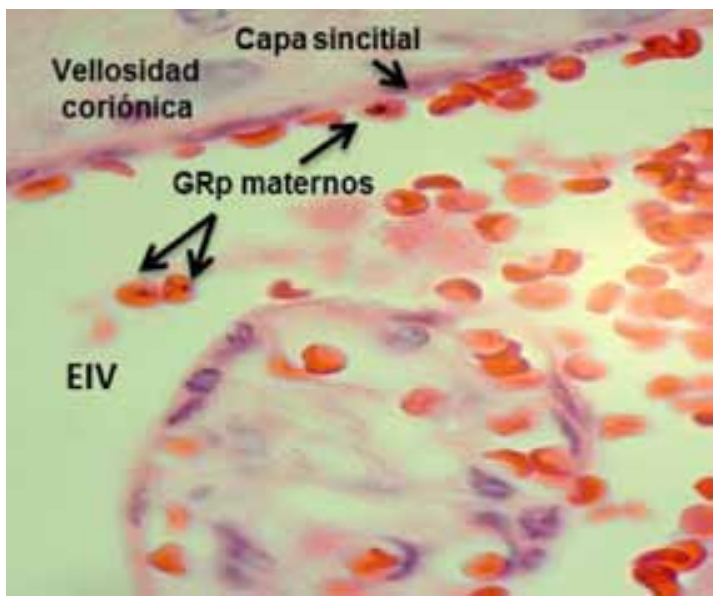

Figura 1. Glóbulos rojos parasitados en el espacio intervelloso. Histopatología, coloración H\&E (100 $X)$ se observa señalado el EIV, lugar en el que se encuentran los glóbulos rojos parasitados (GRp) de la madre, y se identifica la vellosidad coriónica con la correspondiente capa sincitial formada por células del sincitiotrofoblasto, que la separa del EIV. Fuente: Ana María Vásquez, Grupo Malaria 2007.

de la inflamación local en comparación con placentas sanas. Los hallazgos histopatológicos clave en placentas maláricas fueron hechos por Galbraith y cols en 1980 (22), y Walter y cols en 1981 (23), sus estudios reportaron cambios histopatológicos en el tejido placentario como daño a las vellosidades coriónicas con diferentes grados de severidad desde el EIV hasta el estroma velloso. Los estudios realizados por estos autores refirieron como principales características histopatológicas de las placentas maláricas: 1 . Elevación en el infiltrado de monocitos en el EIV; 2. Daño inespecífico al troblasto; 3. Adelgazamiento de la membrana basal del trofoblasto. Posteriormente, en 1989 Yamada y cols (24), reportaron además necrosis focal, pérdida parcial de las microvellosidades, depósitos de fibrina y nodos sincitiales que son agregados de nucleos sincitiales protruyendo hacia el EIV (16).

Todas las alteraciones que afectan la arquitectura de la placenta se derivan de la marcada inflamación local debida a la presencia del parásito. El daño tisular se acompaña de un suministro deficiente de oxígeno al tejido placentario y como consecuencia, se tiene un pobre tránsito de nutrientes y oxígeno en la interfase materno fetal, eventos 


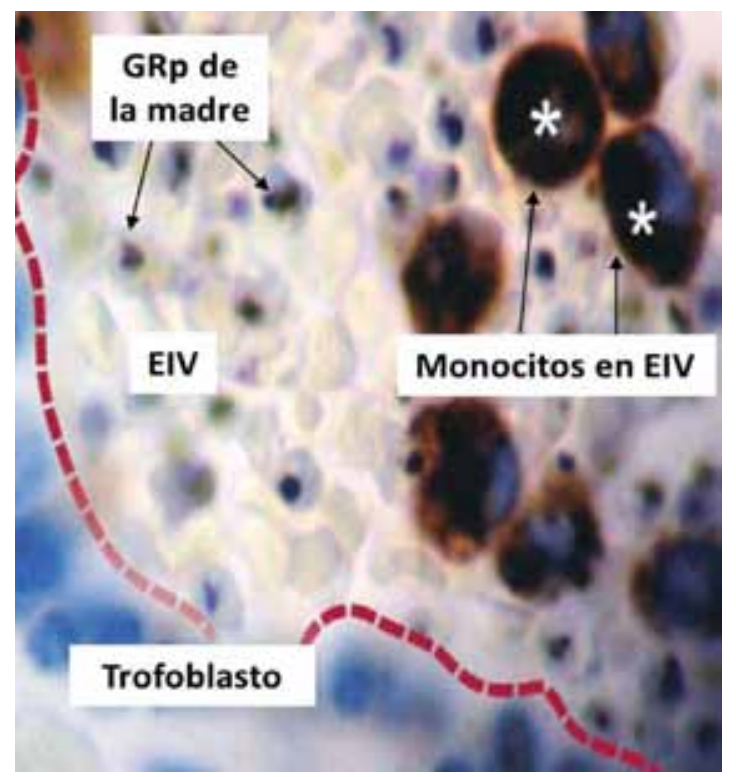

Figura 2. Infiltrado inflamatorio placentario en la infección por Plasmodium spp. Histopatología, coloración inmunohistoquímica $(100 \mathrm{X})$ se observa señalado el espacio intervelloso (EIV), glóbulos rojos parasitados (GRp) de la madre, se identifica el trofoblasto, y células del infiltrado inflamatorio correspondientes a monocitos, en el EIV. Tomado y modificado de: Malaria in pregnancy: pathogenesis and immunity; Stephen J. Rogerson, 2007.

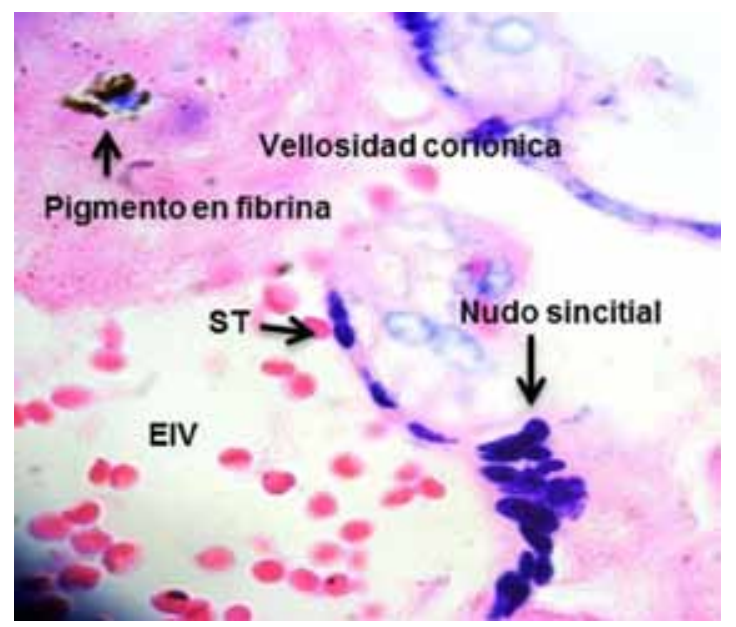

Figura 3. Daño al tejido placentario durante la infección por Plasmodium spp. Histopatología, coloración Giemsa $(100$ X) se observa señalado el EIV, lugar en el que se encuentran los glóbulos rojos parasitados (GRp) de la madre, y se identifica la vellosidad coriónica con depósitos de fibrina y en ella pigmento malárico, adicionalmente la formación de nudos sincitiales. Fuente: Ana María Vásquez, Grupo Malaria 2007. asociados con bajo peso al nacer (BPN) y parto pretérmino (25-27) (Figura 2 y 3).

\section{Glicosaminoglicanos, citoadherencia e inflamación}

Los glicosaminoglicanos (GAGs) son carbohidratos, conocidos tambien como glicanos, organizados en cadenas de heteropolisacáridos generalmente compuestas de hexosamina y ácido glucurónico, los GAGs, normalmente se encuentran unidos a una proteína core dando lugar a la formación de proteoglicanos, ampliamente distribuidos en los tejidos animales $(28,29)$.

En la placenta humana, estas moléculas son producidas por las células del estroma velloso, células del trofoblasto $y$ del sincitiotrofoblasto (30), donde cumplen funciones reguladoras para el sostenimiento de la gestación y, durante la infección por Plasmodium spp, median el secuetro de eritrocitos parasitados en el EIV.

Por lo anterior, la presencia de GAGs en la placenta constituye un aspecto clave en la patología de la placenta malárica $(12,31)$. Uno de los principales glicanos presentes en la placenta, identificado como el receptor por excelencia de eritrocitos parasitados con Plasmodium spp en este tejido es el CSA. En un estudio realizado por Vásquez y cols (32), mencionan que el CSA podría estar actuando indirectamente como inductor de inflamación de la placenta en MP por P. falciparum y otros estudios recientes ponen de manifiesto la participación de esta molécula como regulador de la inflamación en diversos modelos biológicos tales como la enfermedad de Bowel y reparación de diversos tejidos lesionados $(33,34)$.

Además del CSA, en la placenta se presentan otros glicanos como el HA y el HS. El HA, compuesto por cadenas de unidades de disacáridos repetidos de $\mathrm{N}$-acetilglucosamina (GlcNac) y ácido glucurónico, este glicano es particularmente abundante en el endotelio y en la superficie del sincitiotrofoblasto donde se cree que media el sostenimiento de la gestación al promover la proliferación, migración e invasión celular $(35,36)$. Además, en otros tejidos como el pulmón, se ha comprobado su actividad regulatoria de la inflamación durante procesos infecciosos o de injuria, ya que participa en el reclutamiento de células inflamatorias y la posterior liberación de citocinas proinflamatorias (37). EI HA difiere de otros glicanos, en que carece de sulfatación; no obstante, hay pruebas de que es una molécula mediadora del secuestro de eritrocitos parasitados con Plasmodium spp en la placenta $(31,38)$. No se debe perder de vista que en una investigación realizada por Maharjan y cols en 2011 (39), 
en tejido pulmonar, mostró que durante eventos de injuria al tejido o durante procesos infecciosos, el $\mathrm{HA}$ se rompe dando origen a una variedad de HA llamada ácido hialurónico de baja masa molecular (LMWHA), el cual demostró ser promotor de la fagocitosis al estimular particularmente monocitos infiltrados al tejido (40).

Por ultimo, respecto al HS se sabe que es mediador en la formación de rosetas de eritrocitos parasitados así como de la adherencia de eritrocitos parasitados en el endotelio vascular de órganos como el pulmón y el cerebro $(38,41)$, pero no se ha descrito como participe en el secuestro placentario. Sin embargo, un posible rol de esta molécula en la placenta tiene que ver con la modulación de la inflamación local tanto durante la gestación sana como durante la infección placentaria por Plasmodium spp, pues hay estudios en líneas celulares esplénicas que lo involucran de manera contundente como modulador de la respuesta inmune tipo Th1 promoviendo la liberación de citocinas pro-inflamatorias como el TNF- $\alpha$ de gran importancia durante la infección placentaria por Plasmodium spp $(3,4)$.

Queda planteada la necesidad de diseñar estudios que permitan saber cuál es el verdadero rol de los GAGs en los eventos inflamatorios durante la MP y si otras moléculas presentes en el microambiente placentario, influyen en la expresión de estas moléculas.

\section{CONCLUSIÓN}

Los GAGs como moléculas adherentes y reguladoras intervienen sin lugar a dudas en importantes eventos que promueven y sostienen la gestación, identificar con exactitud su participación en la respuesta inflamatoria placentaria en la infección de la placenta por Plasmodium spp no solo permitirá comprender a fondo la fisiopatología de la MP, sino que también permitirá desarrollar estrategias para combatirla oportunamente.

\section{REFERENCIAS}

1. Bakheit $\mathrm{KH}$, Bayoumi NK, Eltom AM, Elbashir MI, Adam I. Cytokines profiles in Sudanese women with preeclampsia. Hypertens Pregnancy 2009;28(2):2249.

2. Fried M, Duffy PE. Maternal malaria and parasite adhesion. J Mol Med (Berl) 1998;76(3-4):162-71.

3. Wrenshall L. Role of the microenviroment in immune response to transplantation. Springer Semin Immunopathol 2003;(2):199-213.

4. Rashid RM, Lee JM, Fareed J, Young MRI. In vitro heparan sulfate modulates the immune responses of normal and tumor-bearing mice. Immunol Invest
2007;36(2):183-201.

5. Suguitan AL, Leke RGF, Fouda G, Zhou A, Thuita L, Metenou S, et al. Changes in the levels of chemokines and cytokines in the placentas of women with Plasmodium falciparum malaria. J Infect Dis 2003 1;188(7):1074-82.

6. Abrams ET, Brown H, Chensue SW, Turner GDH, Tadesse $E$, Lema VM, et al. Host response to malaria during pregnancy: placental monocyte recruitment is associated with elevated beta chemokine expression. $\mathrm{J}$ Immunol 2003;170(5):2759-64.

7. Fried M, Muga RO, Misore a O, Duffy PE. Malaria elicits type 1 cytokines in the human placenta: IFNgamma and TNF-alpha associated with pregnancy outcomes. J Immunol 1998;160(5):2523-30.

8. Jiménez J. Epidemiología de la malaria durante el embarazo. medicasuis.org 2008;(52). Citado 2014 Junio 17. Disponible en: http://www.medicasuis.org/ anteriores/volumen21.3/3.pdf

9. Desai $M$, ter Kuile FO, Nosten F, McGready R, Asamoa K, Brabin B, et al. Epidemiology and burden of malaria in pregnancy. Lancet Infect Dis 2007 Feb;7(2):93-104.

10. Ismail MR, Ordi J, Menendez C, Ventura PJ, Aponte $\mathrm{JJ}$, Kahigwa E, et al. Placental pathology in malaria: a histological, immunohistochemical, and quantitative study. Hum Pathol 2000;31(1):85-93.

11. Steketee RW, Nahlen BL, Parise ME, Menendez C. The burden of malaria in pregnancy in malaria-endemic areas. Am J Trop Med Hyg 2001;64(1-2 Suppl):28-35.

12. Fried M, Duffy $P$. Adherence of Plasmodium falciparum to chondroitin sulfate $A$ in the human placenta. Science 1996;1996:272:502-4.

13. O'Neil-Dunne I, Achur RN, Agbor-Enoh ST, Valiyaveettil M, Naik RS, Ockenhouse CF, et al. Graviditydependent production of antibodies that inhibit binding of Plasmodium falciparum-infected erythrocytes to placental chondroitin sulfate proteoglycan during pregnancy. Infect Immun 2001;69(12):7487-92.

14. Taylor DW, Zhou A, Marsillio LE, Thuita LW, Leke $\mathrm{EB}$, Branch $\mathrm{O}$, et al. Antibodies that inhibit binding of Plasmodium falciparum-infected erythrocytes to chondroitin sulfate $A$ and to the $C$ terminus of merozoite surface protein 1 correlate with reduced placental malaria in Cameroonian women. Infect Immun 2004;72(3):1603-7.

15. Brabin BJ. An analysis of malaria in pregnancy in Africa. Bull World Health Organ 1983;61(6):1005-16.

16. Matteelli A, Caligaris S, Castelli F, Carosi G. The placenta and malaria. Ann Trop Med Parasitol 1997;91(7):803-10.

17. Salih MM, Mohammed AH, Mohmmed AA, Adam GK, Elbashir MI, Adam I. Monocytes and macrophages and placental malaria infections in an area of unstable malaria transmission in eastern Sudan. Diagn Pathol 2011;6(1):83. doi: 10.1186/1746-1596-6-83

18. Rico-Rosillo M, Robledo G. Mecanismos inmunológicos involucrados en el embarazo. Ginecol Obs Mex 2012;80(5):332-40.

19. Bardají A, Sigauque B, Sanz S, Maixenchs M, Ordi 
$\mathrm{J}$, Aponte JJ, et al. Impact of malaria at the end of pregnancy on infant mortality and morbidity. $\mathrm{J}$ Infect Dis 2011;203(5):691-9.

20. Brabin BJ, Romagosa C, Abdelgalil S, Menéndez C, Verhoeff FH, McGready R, et al. The sick placenta-the role of malaria. Placenta 2004;25(5):359-78.

21. Maubert B, Fievet N, Tami G, Boudin C, Deloron P. Cytoadherence of Plasmodium falciparum-infected erythrocytes in the human placenta. Parasite Immunol 2000;22(4):191-9.

22. Galbraith RM, Fox H, Hsi B, Galbraith GM, Bray RS, Faulk WP. The human materno-foetal relationship in malaria. II. Histological, ultrastructural and immunopathological studies of the placenta. Trans R Soc Trop Med Hyg 1980;74(1):61-72.

23. Walters PR, Garin Y, Philippe B. Placental pathologic changes in malaria placental pathologic changes in malaria a histologic and ultrastructural study. Am Assoc Pathol 1982;109:330-42.

24. Yamada M, Steketee R, Abramowsky C, Kida M, Wirima J, Heymann D, Rabbege J, Breman J, Aikawa M. Plasmodium falciparum associated placental pathology: a light and electron microscopic and immunohistologic study. Am J Trop Med Hyg 1989;41:161-8.

25. Rogerson SJ, Brown HC, Pollina E, Abrams ET, Tadesse E, Lema VM, Molyneux ME. Placental tumor necrosis factor alpha but not gamma interferon is associated with placental malaria and low birth weight in Malawian women. Infect Immun 2003;71(1):267-70.

26. Bulmer JN, Rasheed FN, Francis N, Morrison L, Greenwood BM. Placental malaria. I. Pathological classification. Histopathology 1993;22(3):211-8.

27. McGready R, Davison BB, Stepniewska K, Cho T, Shee $\mathrm{H}$, Brockman $\mathrm{A}$, et al. The effects of Plasmodium falciparum and $P$. vivax infections on placental histopathology in an area of low malaria transmission. Am J Trop Med Hyg 2004;70(4):398-407.

28. Dwek MV, Brooks SA, Schumacher U. Functional \& Molecular Glycobiology. BIOS Scientific 2002.

29. Gagneux P, Varki A. Evolutionary considerations in relating oligosaccharide diversity to biological function. Glycobiology 1999;9(8):747-55.

30. Kirn-Safran C, D'Souza S, Carson D. Heparan sulfate proteoglycans and their binding proteins in embryo implantation and placentation. Semin Cell Dev Biol 2008;19(2):187-93.
31. Beeson J, Rogerson S, Cooke B. Adhesion of Plasmodium falciparum-infected erythrocytes to hyaluronic acid in placental malaria. Nat Med 2000;6(1):86-90.

32. Vásquez AM, Segura C, Blair S. Induction of proinflammatory response of the placental trophoblast by Plasmodium falciparum infected erythrocytes and TNF. Malar J 2013;12:421. doi: 10.1186/1475-287512-421.

33. Principi M, Giorgio F, Losurdo G, Neve V, Contaldo A, Di Leo A, et al. Fibrogenesis and fibrosis in inflammatory bowel diseases: Good and bad side of same coin? World J Gastrointest Pathophysiol 2013;4(4):100-7.

34. Thelin MA, Bartolini B, Axelsson J, Gustafsson R, Tykesson E, Pera E, et al. Biological functions of iduronic acid in chondroitin/dermatan sulfate. FEBS $\mathrm{J}$ 2013;280(10):2431-46. Available from:

35. Sunderland CA, Bulmer JN, Luscombe M, Redman CW SG. Immunohistological and biochemical evidence for a role for hyaluronic acid in the growth and development of the placenta. J Reprod Immunol 1985;8:197-212.

36. Parmley RT, Takagi M DF. Ultrastructural localization of glycosaminoglycans in human term placenta. Anat Rec 1984;210(3):477-84.

37. Noble PW1, Albera C, Bradford WZ CU. Pirfenidone in patients with idiopathic pulmonary fibrosis (CAPACITY): two randomised trials. Lancet 2011;377(9779):1760-9.

38. Beeson JG, Rogerson SJ, Brown G V. Evaluating specific adhesion of Plasmodium falciparum-infected erythrocytes to immobilised hyaluronic acid with comparison to binding of mammalian cells. Int $\mathrm{J}$ Parasitol 2002;32(10):1245-52.

39. Maharjan AS, Pilling D, Gomer RH. High and low molecular weight hyaluronic acid differentially regulate human fibrocyte differentiation. PLoS One 2011;6(10):e26078.

40. Ismail MR, Ordi J, Menendez C, Ventura PJ, Aponte JJ, Kahigwa E, et al. Placental pathology in malaria: a histological, immunohistochemical, and quantitative study. Hum Pathol 2000;31(1):85-93.

41. Chen $Q$, Barragan $A$, Fernandez V, Sundström $A$, Schlichtherle M, Sahlén A, Carlson J, Datta S, Wahlgren $M$. Identification of Plasmodium falciparum erythrocyte membrane protein 1 (PfEMP1) as the rosetting ligand of the malaria parasite $P$. falciparum. $J$ Exp Med 1998;187(1):15-23. 